\title{
MA-KUAGRO: ELEMENTO DE LA CULTURA PALENQUERA Y SU INCIDENCIA EN LAS PRÁCTICAS PEDAGÓGICAS EN LA ESCUELA SAN BASILIO PALENQUE, COLOMBIA
}

Dimas Del Rosario de Ávila Torres ${ }^{[1]}$ Rutsely Simarra Obeso ${ }^{[2]}$

\section{Resumen}

La investigación analizó los elementos de la cultura palenquera presentes en la escuela, particularmente "kuagro"3 que es manifestación organizativa propia, y su vínculo con las prácticas pedagógicas del personal docente y el aprovechamiento académico estudiantil. Este estudio aporta al proceso etno-educativo llevado a cabo en San Basilio de Palenque y otras comunidades culturalmente diferenciadas en Colombia. La investigación fue de tipo cualitativo, se hizo uso del método de la consulta a la memoria colectiva, creado por la comunidad palenquera en el marco del proceso de etno-educación. Las técnicas y herramientas fueron la observación, entrevista y conversatorios con docentes, estudiantes, líderes y lideresas, miembros del consejo comunitario.

Entre los resultados se menciona que existe una articulación en la Institución Educativa Benkos Biohó y la comunidad, ya que el acervo cultural es el eje principal de las prácticas pedagógicas. Entre estos, se ha incluido los patios productivos, las ceremonias fúnebres, la música, entre otros, con la participación de la gente anciana y sabia de la comunidad. Sin embargo, a pesar de lo anterior, los saberes y prácticas pedagógicas son diversos desde la perspectiva de los docentes sensibilizados, no sensibilizados y los que están en proceso de sensibilización. Los docentes sensibilizados han logrado un mejor desempeño académico en los estudiantes.

Palabras Clave: Etno-educación, palenquera, Kuagro, pedagogías propias.

\footnotetext{
${ }^{[1]}$ Máster en Educación Intercultural Multilingüe, etnoeducadora - investigadora en Cartagena, Colombia. rosariodeavila@hotmail.com

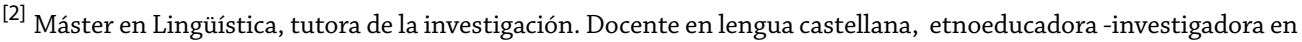
Cartagena, Colombia. rutselysimarraster@gmail.com

${ }^{[3]}$ Grupos organizados por edad que se establecen desde la infancia y se pueden considerar como los espacios de socialización y organización social de los palenqueros.
} 


\section{Summary}

The research analyzed the elements of the palenque culture that are present in the school, particularly "kuagro" that it's his own organizational manifestation, and its link with the teachers pedagogical practices and student achievement. This study contributes to the ethno-educational process conducted in San Basilio de Palenque and other communities in Colombia that are culturally different. The research was qualitative; we made use of the method of consulting the collective memory, created by the palenque community in the framework of the ethno-education process. The techniques and tools that guided the investigation were: the observation, interviews and conversations with teachers, students, women and men leaders, as well as with the members of the community council.

Among the results we can highlight that there is an articulation between the Benkos Bioho educational institution and the community, being that cultural heritage is the axis of the teaching practices. Among these, we have included the productive yards, funeral ceremonies, music, among others, with the participation of the wise and elderly people in the community. However, despite this, the knowledge and pedagogical practices are different from the perspective of sensitized teachers, nonsensitized and those in processes of sensitization. Sensitized teachers have achieved a better academic performance in students.

Keywords: Ethno-education, Palenque, Kuagro, self-pedagogies.

\section{Introducción}

La presente investigación evidencia la vinculación de $\mathrm{Ma}^{\text {- }}$ kuagro ${ }^{4}$ en la escuela, principalmente en las prácticas pedagógicas del personal docente de la Institución Educativa Técnica Agropecuaria Benkos Biohó de San Basilio de Palenque. De igual forma, la apropiación de los elementos culturales para el aprovechamiento académico.

Este estudio aporta al proceso etnoeducativo que se lleva a cabo en Palenque y otras comunidades y pueblos culturalmente diferenciados de Colombia. Decir etnoeducación, para el caso colombiano es referirse a la Educación Intercultural Bilingüe y/o multilingüe como se denomina en otros países de Suramérica y Centroamérica.

La comunidad en donde se llevó a cabo la investigación es San Basilio de Palenque, es una comunidad fundada por los esclavizados que se fugaron y se refugiaron en los palenques de la Costa Caribe de Colombia desde el siglo XV. El término palenque se

\footnotetext{
${ }^{[4]}$ Ma Kuagro en lengua palenquera corresponde al conjunto de Kuagros en plural.
} 
define como aquel lugar poblado por cimarrones o esclavizados africanos fugados del régimen esclavista durante el período colonial. De ahí que se convirtió en sinónimo de libertad, ya que toda persona que llegaba a formar parte de un palenque era automáticamente libre. Guerrero, Hernández, J.P. Pérez J, N, Pérez, Restrepo.(2004)

De los innumerables palenques conformados en la época de la colonia para el caso de Colombia, hoy pervive San Basilio, que lucha por preservar sus elementos culturales e identitarios.

\section{Revisión de literatura}

\section{Etnoeducación}

Para el caso de Colombia y según D. Hernández, Simarra, R. Hernández. (2004), etnoeducación es entendida como:

Un proceso social permanente de reflexión y construcción colectiva, mediante la cual los pueblos indígenas, afrocolombianos y rom o gitanos fortalecen su autonomía en el marco de la interculturalidad, posibilitando la interiorización y producción de valores, de conocimientos y el desarrollo de habilidades y destrezas conforme a su realidad cultural, expresada en su proyecto global de vida.

Esta propuesta pedagógica alternativa está basada en los siguientes principios, según Hernández et al (2004), en la identidad, autonomía, interculturalidad, solidaridad, integralidad, reflexibilidad y progresividad.

\section{Pedagogías propias}

De acuerdo con Aguilar M. \& Rodríguez M. (1999), las pedagogías propias se muestran:

Como una alternativa pedagógica que ayuda a despejar el camino en cuanto al desarrollo de unas metodologías apropiadas, al abordar las problemáticas particulares de las comunidades negras del país; si bien no se plantea como una experiencia única, sí es el resultado de un largo trabajo de construcción e indagación que puede dar luces a otro tipo de experiencias que se realicen en el país.

Con base en lo anterior, las pedagogías propias son aquellas prácticas de enseñanzaaprendizaje que tradicionalmente usa un pueblo culturalmente diferenciado para transmitir oralmente conocimientos de generación en generación. 
La escuela debe apropiarse de estas formas de enseñanzas para facilitar y potencializar las habilidades y competencias de sus estudiantes, generando con ello una interrelación entre la escuela y la comunidad.

\section{kuagro}

La historia del kuagro está relacionada con el proceso de cimarronaje y todos los mecanismos de resistencia empleados por los esclavizados en el siglo XVI. Desde la captura en tierras africanas y el largo viaje en los barcos negreros en condiciones infrahumanas.

De acuerdo con Sánchez y Patiño (1983), en vernáculo, el kuagro son grupos que se definen como asociaciones basadas en la edad, ingresan hombres y mujeres que permanecen allí por el resto de su vida. Su membrecía se afirma cuando sus individuos llegan a la pubertad.

Según Guerrero, C. I.; Hernández, R. y Pérez, J. (2008), kuagro en San Basilio de Palenque son grupos organizados por edad, que se establecen por generaciones desde la infancia y se pueden considerar como los espacios de socialización secundaria donde se condensa la organización social de este grupo étnico.

En el kuagro confluyen todas las expresiones y elementos de esta comunidad, es donde se ponen en práctica las interacciones y representaciones sociales, las contradicciones y la resolución de conflictos y todos los aspectos que tengan que ver con la vida misma de este colectivo social. A través de él se recrea la lengua palenquera, los rituales fúnebres, las prácticas tradicionales de producción, la cuentería, la medicina tradicional y los demás elementos culturales.

Esto se evidencia en la dinámica organizativa del kuagro donde la tendencia es que la gente del kuagro, se mantengan hasta la muerte con su colectivo. Sin embargo, puede ocurrir que un miembro del kuagro cambie a otro por razones personales.

\section{Escuela y cultura}

De acuerdo a Jerome Brunner (1997), "No se puede comprende al hombre sin entender la cultura en la que está inserto. Por lo tanto, la escuela está en la obligación de entender el contexto donde desarrolla su praxis, no sólo para traer a la escuela las diferentes manifestaciones culturales, como la lengua, rituales fúnebres, danzas, música y todo el acervo cultural que caracteriza determinado territorio, también para analizar los aspectos de la cultura que pueden contribuir con la formación personal de los estudiantes.

Es decir, prepararlos para el saber, el hacer y el ser. Desde su cosmovisión pero llevándolos a las relaciones interculturales que faciliten el respeto y la aceptación de 
las diferencias de cualquier tipo. Es así que la cultura es determinante en todas las dimensiones de desarrollo de sus integrantes, la manera de conformar la familia, las relaciones entre sus miembros, solución de conflictos, y por ende la forma como se educan sus miembros, donde la educación debe responder a la forma de vida de cada pueblo.

\section{Materiales y métodos}

La investigación realizada es de tipo cualitativo, se hizo uso del método de la consulta a la memoria colectiva, método creado por la comunidad palenquera en el marco del proceso de etnoeducación. En este método de investigación, la propia comunidad es considerada sujeto, objeto y fuente de investigación. Las técnicas y herramientas de investigación en el trabajo de campo fueron la observación, entrevista, conversatorio con docentes, estudiantes, líderes y lideresas, miembros del consejo comunitario que aportaron sus saberes acerca del tema en mención.

Las unidades de análisis fueron los docentes y la rectoría, así como los estudiantes. Además se realizaron conversatorios con líderes y lideresas de diferentes organizaciones comunitarias.

Entre los principales criterios de inclusión de los actores claves del estudio fueron:

- Pertenecer en calidad de docente o estudiante a la Institución educativa Benkos Biohó.

- Maestros y estudiantes conocedores e impulsores del fortalecimiento y preservación de las manifestaciones culturales palenqueras.

- Maestros con más de cinco años laborando en la institución y que hayan estado activos en los procesos de resignificación del Proyecto Educativo Institucional. (P.E.I.).

- Estudiantes que pertenezcan en la actualidad a un Kuagro palenquero.

Finalmente, el procesamiento y análisis se realizó mediante el diseño de una matriz de descriptores diseñada desde el protocolo de la investigación. 


\section{Resultados y discusión}

\section{El proceso etnoeducativo en la comunidad de Palenque}

De acuerdo con Aguilar Rodríguez (1999), la comunidad de Palenque ha desarrollado estrategias de supervivencia cultural que han garantizado la transmisión de la lengua, la religión y los demás valores. Entre estas estrategias se concibe la educación como uno de los vehículos fundamentales de transmisión y protección cultural.

De acuerdo al Observatorio Del Caribe Colombiano (2010), en los años noventa la etnoeducación en Palenque se convirtió en un movimiento cultural activo en donde se realizaban un sin número de eventos de revitalización cultural. Sin embargo, existían sectores sobre todo de profesionales que estaban en desacuerdo porque consideraban que la etnoeducación era un atraso, lo mismo que hablar la lengua y tratar temas de la cultura.

En ese mismo período, se constituyó la oficina de etnoeducación en el Centro Experimental Piloto, posteriormente, la oficina en mención pasó a la Secretaría de Educación Departamental. Lo anterior, permitió valorar la lengua propia, la ritualidad del Lumbalú, el kuagro, la medicina tradicional, la gastronomía, la música y la danza, la autoridad ancestral y comunitaria y un sin número de valores que habían entrado en decadencia.

Este proceso etnoeduativo, pedagógicamente, se organizó de tal manera que un kuagro era como el coordinador de toda la actividad, y se logró estructurar un "kuagro de lengua" un "kuagro sociocultural", un "kuagro de historia" y esta era la forma orgánica del proceso.

Es de pleno conocimiento que un Kuagro es la forma de organización social de Palenque y para ese entonces, estos grupos tenían además entre sus funciones orientar todo el proceso de recuperación y revitalización, por ejemplo, el "kuagro de lengua" constituido en comité permanente de trabajo sesionaba rigurosamente día a día.

San Basilio de Palenque es una comunidad con una gran riqueza cultural que ha sobrevivido a lo largo de tres siglos. Por lo anterior, en el año 2005 la comunidad fue reconocida por la UNESCO como Obra Maestra del Patrimonio Inmaterial de La Humanidad.

\section{Manifestaciones culturales visibilizadas en la Institución Educativa Benkos Biohó}

La articulación escuela y comunidad, inicia con la sensibilización de los líderes comunitarios y líderes educativos, como parte del movimiento etnoeducativo. En este 
particular, se plantea el acervo cultural como eje principal de las prácticas pedagógicas. Así mismo, la participación de los ancianos palenqueros en el proceso de enseñanzaaprendizaje.

Tomando en consideración lo anterior, los patios producticos (huertos caseros) han sido un espacio de trascendencia en San Basilio de Palenque, ya que ancestralmente son el punto de reunión familiar, alrededor de la hornilla y de los animales domésticos. Por tanto, en la relación escuela -cultura en la clase de recursos naturales, se les enseña a los estudiantes del instituto a desarrollar el Gavileno o mano cambia. Esta práctica comunitaria consiste en que los miembros del kuagro se turnan para sembrar o adecuar el terreno de cada miembro, hasta recoger la cosecha.

De igual forma, el docente de Matemáticas desarrolla actividades de medidas en las áreas determinadas para los patios productivos. Por otro lado, la docente de la asignatura de artística usa estos espacios para trabajar planos y dibujos.

En estas actividades se vincula a los ancianos de la comunidad en donde son tomados en cuenta sus saberes y también se convierte en padrino de patio. También se realizan rituales de bautizo, que consiste en regar agua en el lugar de la siembra y hacer unas oraciones para pedirle a los dioses que todo lo sembrado prospere, recuperando así, desde la escuela, la ritualidad palenquera en compañía de los estudiantes y el docente de educación religiosa.

Además de los patios productivos, la intervención de los ancianos en la historia del kuagro ha sido significativa en el aprovechamiento académico. "Con el trabajo colectivo se facilitó la intervención de los mayores narrándoles a los estudiantes la historia de los kuagros, muchos jóvenes desconocían esto a pesar de que pertenecían a un kuagro" (Docente de la Escuela entrevistado).

La intervención de los ancianos en la recuperación de la historia de Ma-kuagro, conllevó a que se entendieran mejor los hechos históricos. De igual forma, ha sensibilizados a los estudiantes al nivel que en su momento, decidieron ponerle a las aulas de clases nombres de los valores que caracterizan al kuagro, o pintar la sillas de los salones en común acuerdo entre sus integrantes.

De igual forma la música de la Cultura Palenquera ha sido llevada a la escuela, mediante los ancianos integrantes del Sexteto Tabalá. Ante ello, el docente Luis Marrugo Fruto, plantea:

Igual sucedió con el sexteto Tabalá agrupación musical palenquera, cuyo ritmo es una mezcla entre el son cubano y el bullerengue típico palenquero, la razón social del sexteto es la de amenizar los velorios tratando que el alma del difunto sea acompañada con las notas musicales lo que permite el trance entre el más allá y el más acá. 
La escuela entiende la importancia de potencializar a los jóvenes con actitudes para la música e inicia el proceso de recuperación de estas manifestaciones con los estudiantes, asesorado por los ancianos integrantes del sexteto.

Se necesitaba un relevo generacional, llevando el sexteto a kuagro de jóvenes que le gustaba la música de sexteto, trabajando con ellos según su inclinación por los instrumentos el de las Maracas, el Bongo (Luis Marrugo, docente).

El sexteto Tabalá juega un papel fundamental en la realización del ritual fúnebre Lumbalú, por lo que algunos docentes sensibilizados con el tema etnoeducativo recuperan y revitalizan esta manifestación afirmando la relación escuela- cultura.

Cada miembro del sexteto trabaja con miembros del kuagro interesados en los diferentes instrumentos, los jóvenes iban a las casa de los integrantes del sexteto, y empezaron los jóvenes en los velorios en primera fila en cualquier momento que un señor se cansaba ellos asumían, el velorio es un espacio pedagógico para la comunidad, es éste un espacio de aprendizaje (Luis Marrugo, docente).

\section{Saberes y prácticas pedagógicas del Ma-kuagro}

Los conceptos de pedagogías propias, etnoeducación, manifestaciones culturales, interculturalidad, educación intercultural deben ser el fundamento principal en las prácticas pedagógicas en San Basilio de Palenque. Sin embargo, en las observaciones de clases realizadas, así como en las entrevistas a los docentes, se pudieron establecer categorías que determinan la práctica pedagógica de los maestros. Entre estos, están los docentes sensibilizados, los no sensibilizados y los sensibilizados que intentan implementar el proceso de educación propia.

\begin{tabular}{|c|c|c|c|}
\hline $\begin{array}{l}\text { Tipo o clases de } \\
\text { docentes }\end{array}$ & \multirow{2}{*}{$\begin{array}{c}\text { Docentes } \\
\text { sensibilizados } \\
\text { con el proyecto } \\
\text { etnoeducativo }\end{array}$} & \multirow{2}{*}{$\begin{array}{l}\text { Docentes no } \\
\text { sensibilizados } \\
\text { con el proyecto } \\
\text { etnoeducativo }\end{array}$} & \multirow{2}{*}{$\begin{array}{c}\text { Docentes } \\
\text { sensibilizados que } \\
\text { intentan acercase } \\
\text { al proyecto } \\
\text { etnoeducativo }\end{array}$} \\
\hline $\begin{array}{l}\text { Desarrollo } \\
\text { del proceso } \\
\text { pedagógico }\end{array}$ & & & \\
\hline $\begin{array}{l}\text { Exploración del } \\
\text { conocimiento }\end{array}$ & $\begin{array}{l}\text { Parten de los } \\
\text { conocimientos previos de } \\
\text { los estudiantes. }\end{array}$ & $\begin{array}{l}\text { Algunas veces parten de } \\
\text { los conocimientos previos } \\
\text { de los estudiantes, otras } \\
\text { no. }\end{array}$ & $\begin{array}{l}\text { nta los } \\
\text { s previos de } \\
\text { s en } \\
\text { ecificas. }\end{array}$ \\
\hline $\begin{array}{c}\text { Prácticas } \\
\text { pedagógicas } \\
\text { teniendo en cuenta } \\
\text { los líderes }\end{array}$ & $\begin{array}{l}\text { Clases activas donde los } \\
\text { portadores de saberes de } \\
\text { la comunidad son agentes } \\
\text { educativos para los estu- } \\
\text { diantes. }\end{array}$ & $\begin{array}{l}\text { Clases magistrales con } \\
\text { poca participación de los } \\
\text { estudiantes sin tener en } \\
\text { cuenta los sabios de la } \\
\text { comunidad. }\end{array}$ & $\begin{array}{l}\text { Clases donde puede } \\
\text { tenerse en cuenta los } \\
\text { sabios de la comunidad } \\
\text { en algunas ocasiones, } \\
\text { como referente para los } \\
\text { estudiantes. }\end{array}$ \\
\hline
\end{tabular}




\begin{tabular}{|c|c|c|c|}
\hline $\begin{array}{c}\text { Tipo o clases de } \\
\text { docentes }\end{array}$ & \multirow{2}{*}{$\begin{array}{c}\text { Docentes } \\
\text { sensibilizados } \\
\text { con el proyecto } \\
\text { etnoeducativo }\end{array}$} & \multirow{2}{*}{$\begin{array}{l}\text { Docentes no } \\
\text { sensibilizados } \\
\text { con el proyecto } \\
\text { etnoeducativo }\end{array}$} & \multirow{2}{*}{\begin{tabular}{|c} 
Docentes \\
sensibilizados que \\
intentan acercase \\
al proyecto \\
etnoeducativo
\end{tabular}} \\
\hline $\begin{array}{l}\text { Desarrollo } \\
\text { del proceso } \\
\text { pedagógico }\end{array}$ & & & \\
\hline $\begin{array}{l}\text { Investigación } \\
\text { Pedagógica }\end{array}$ & $\begin{array}{l}\text { Implementación del } \\
\text { componente investigativo } \\
\text { desde las diferentes áreas } \\
\text { del saber. }\end{array}$ & $\begin{array}{l}\text { Poca investigación desde } \\
\text { el aula, en las diferentes } \\
\text { áreas. }\end{array}$ & $\begin{array}{l}\text { Se investiga en algunos } \\
\text { temas específicos desde } \\
\text { el aula en las diferentes } \\
\text { áreas. }\end{array}$ \\
\hline $\begin{array}{c}\text { Relación } \\
\text { comunidad-escuela }\end{array}$ & $\begin{array}{l}\text { Aprendizaje significativo } \\
\text { a partir de lo propio a lo } \\
\text { general, por estar media- } \\
\text { do por un proyecto edu- } \\
\text { cativo comunitario. }\end{array}$ & $\begin{array}{l}\text { Poco interés por el apren- } \\
\text { dizaje, sin mediación de } \\
\text { proyecto educativo comu- } \\
\text { nitario. }\end{array}$ & $\begin{array}{l}\text { Algunas temáticas muy } \\
\text { impactante para los estu- } \\
\text { diantes otras no, algunas } \\
\text { veces mediadas por el } \\
\text { proyecto educativo comu- } \\
\text { nitario. }\end{array}$ \\
\hline $\begin{array}{c}\text { Relaciones entre } \\
\text { docentes y } \\
\text { estudiantes }\end{array}$ & $\begin{array}{l}\text { Relaciones entre docentes } \\
\text { y estudiantes en línea } \\
\text { horizontal. }\end{array}$ & $\begin{array}{l}\text { Relaciones entre docentes } \\
\text { y estudiantes en línea } \\
\text { vertical. }\end{array}$ & $\begin{array}{l}\text { Relaciones entre docentes } \\
\text { y estudiantes en línea } \\
\text { vertical. }\end{array}$ \\
\hline $\begin{array}{l}\text { Aprehensión del } \\
\text { conocimiento }\end{array}$ & $\begin{array}{l}\text { Mayor empoderamiento } \\
\text { de los conocimientos por } \\
\text { parte de los estudiantes. }\end{array}$ & $\begin{array}{l}\text { Los conocimientos adqui- } \\
\text { ridos puedan olvidarse } \\
\text { fácilmente. }\end{array}$ & $\begin{array}{l}\text { Algunos conocimientos } \\
\text { perduran, otros pueden } \\
\text { olvidarse fácilmente. }\end{array}$ \\
\hline $\begin{array}{c}\text { Aprovechamiento } \\
\text { académico }\end{array}$ & $\begin{array}{l}\text { Estudiantes con mejor } \\
\text { desenvolvimiento en el } \\
\text { proceso lector y escritor. }\end{array}$ & $\begin{array}{l}\text { Estudiantes con algunas } \\
\text { dificultades en el proceso } \\
\text { lector y escritor. }\end{array}$ & $\begin{array}{l}\text { Estudiantes con algunas } \\
\text { facilidades en el proceso } \\
\text { lector y escritor. }\end{array}$ \\
\hline $\begin{array}{c}\text { Prácticas docentes } \\
\text { efectivas }\end{array}$ & $\begin{array}{l}\text { Docentes que confían en } \\
\text { sus estudiantes lleván- } \\
\text { dolos a alcanzar logros } \\
\text { académicos. }\end{array}$ & $\begin{array}{l}\text { Docentes que no consi- } \\
\text { deran a los estudiantes } \\
\text { sujetos de cambios. }\end{array}$ & $\begin{array}{l}\text { Docentes que reconocen } \\
\text { algunos estudiantes con } \\
\text { capacidad para alcanzar } \\
\text { algunos logros acadé- } \\
\text { micos. }\end{array}$ \\
\hline $\begin{array}{c}\text { Todos los actores } \\
\text { del proceso } \\
\text { pedagógico portan } \\
\text { saberes }\end{array}$ & $\begin{array}{l}\text { El maestro y el estudiante } \\
\text { son portadores de sa- } \\
\text { beres. }\end{array}$ & $\begin{array}{l}\text { El maestro es quien porta } \\
\text { el saber. }\end{array}$ & $\begin{array}{l}\text { En algunas actividades se } \\
\text { reconoce el saber de los } \\
\text { estudiantes. }\end{array}$ \\
\hline
\end{tabular}

El cuadro anterior, define por un lado avances en el proceso etnoeducativo, pero también plantea los principales desafíos de este proceso. Sin embargo, a pesar de los esfuerzos del movimiento social afrocolombiano, los docentes entrevistado plantean que todavía faltan políticas públicas para la autoeducación y por ende existe poca voluntad política por parte del estado, de tal forma que se evidencie la educación de los grupos étnicos.

Entre estas políticas públicas, está la poca formación en etnoeducación de los directivos y docentes, de tal forma que les permita implementar y direccionar estrategias pedagógicas pertinentes. Basado en lo anterior, los docentes plantean lo siguiente: 
El proceso puede avanzar en algunos compañeros, pero en lo que son radicales no permiten avanzar, la rectora si bien no es de Palenque, tiene poca claridad ante el tema, no dice no a la propuesta, pero tampoco aporta. Deja que los procesos se desarrollen (Luis Rodríguez, Docente de Ciencias Sociales).

En relación al desempeño académico de los estudiantes, los docentes que han implementado la propuesta pedagógica etnoeducativa, teniendo en cuenta el kuagro han logrado mejores resultados. Es decir, experiencias como los patios productivos, la recuperación de las manifestaciones musicales a través de sexteto, el ritual fúnebre Lumbalú y el kuagro de niñas rezanderas han conducido a los estudiantes a una mayor capacidad de análisis de las situaciones de la comunidad y de otros contextos.

Así lo evidencia el estudiante Adonis Herrera Pérez, quien afirma que: "Los docentes nos reúnen teniendo en cuenta los Kuagros, esto nos permite ayudarnos mutuamente". Los estudiantes entrevistados aseguran que casi todos pertenecen a kuagro, de igual forma, les es satisfactorio que sus que sus maestros tengan en cuenta los elementos culturales, que les permita ser cada día mejores estudiantes.

\section{Conclusiones}

El trabajo investigativo permitió un mayor acercamiento con la comunidad de San Basilio de Palenque y sus manifestaciones culturales específicamente Ma-kuagro.

Ma-Kuagro es la esencia de la vida de la cultura palenquera, es la célula organizativa fundamental, donde se fortalecen los valores que permiten interactuar en sociedad; sin embargo, la escuela necesita mayor interacción con estas manifestaciones culturales y poner a la orden de las prácticas pedagógicas todo el acervo cultural palenquero.

Se pudo identificar con la realización de la investigación las prácticas pedagógicas empleadas por los docentes de la Institución Educativa Técnica Agropecuaria Benkos Biohó, y su vinculación con el acervo cultural. En este sentido, existen diferentes niveles de aplicación tanto en docentes sensibilizados, no sensibilizado y los que están en proceso de sensibilización.

Si bien es cierto que el esfuerzo de algunos docentes por desarrollar actividades pedagógicas teniendo en cuenta el acervo cultural se ve reflejado en el aprovechamiento académico, se necesita que todos y cada uno de éstos se comprometan realmente con el proyecto o plan de vida comunitario, que se facilite a través de la escuela y sus prácticas pedagógica en el etnodesarrollo o buen vivir desde la cosmovisión palenquera. 


\section{REVITALIZACIÓN LINGÜÍSTICA Y CULTURAL}

\section{Lista de referencias}

Aguilar Muñoz, S. \& Rodríguez Manotas L.F. (1999). Tradición oral y pedagogía propias. En: Culturas, lenguas y educación: Simposio de Etnoeducación, VIII Congreso de Antropología. Memorias. Bogotá: Universidad de los Andes.

Arrázola R. (200o). Palenque, primer pueblo libre de América. Cartagena: Ediciones Hernández.

Cassiani, A. (2002). San Basilio de Palenque: historia de la resistencia, 1599-1713.En: 150 años de la abolición de la esclavización en Colombia. Desde la marginalidad a la construcción de la nación. Bogotá.

Cerda, H. (2002). Los elementos de la investigación. Bogotá, El Búho.

Dieck, M. (1998). Criollistica Afrocolombiana. En: Geografía humana de Colombia. Los Afrocolombianos. Tomo IV. Bogotá: Instituto Colombiano de Cultura Hispánica Santa Fe de Bogotá. D.C.

Dossier (2002). Palenque de San Basilio Obra Maestra del Patrimonio Intangible de la Humanidad. Ministerio de Cultura, Presidencia de la república. Palenque-Bogotá,

Friedmann, N. S. \& Patiño Rosselli, C.(1983). Lengua y sociedad en el Palenque de San Basilio. Bogotá, Instituto Caro y Cuervo.

Friedmann, N. S. \& Richard Cross (1979). Ma Ngombe. Guerreros y ganaderos en Palenque. Bogotá, Carlos Valencia Editores.

García Sánchez, Andrés (2003). Fiestas y celebraciones en Palenque. En: Ramiro Delgado (coord.), Etnografías y patrimonios. Relatos de san Basilio de Palenque. Comunidad de San Basilio de Palenque- Fondo del Patrimonio Cultural de la Embajada de los estados Unidos- Medellín: Universidad de Antioquia.

Guerrero, C. I.; Hernández, R. y Pérez, J. (2008). Palenque: Historia libertaria, cultura y tradición. Cartagena: Grupo de Investigación Muntú.

Guerrero G, C. I. Hernández Cassiani, R. \& Pérez Palomino, J. (2008). Palenque: Historia libertaria, cultura y tradición. 
Guía Para El Diseño y Presentación De Protocolos De Investigación (2008). Universidad De Las Regiones Autónomas De La Costa Caribe Nicaragüense.

Hernández Cassiani, R. (2009). Movimientos sociales, identidad y sujetos de poder: una revisión de las prácticas pedagógicas comunitarias y su incidencia en el desarrollo de las comunidades afrodescendientes. Por publicar. 